Methods: A retrospective study including all patients suffering from $\mathrm{CHC}$ followed over a period of 11 years (2002-2012). Were excluded all patients co-infected by hepatitis $B$ virus or by human immunodefficiency virus and those having decompensated cirrhosis. Different RM were collected and analysed according to the epidemiological, clinico-biological, immunological, virological and histological data of the $\mathrm{CHC}$.

Results: Two hundred and four patients affected by $\mathrm{CHC}$ were included, meanly aged by 52 years [22-66 years]. The sex-ratio was 0,46 . MR were noted in 76 patients $(37,25 \%)$ dominated by inflammatory polyarthralgia of big joints $(88,25 \%)$. Non erosive arthritis was observed in a woman and was localised to the proximal interphalangeal articulation of the index. Myalgia were noted in 11 cases $(14,47 \%)$ among them, 2 appeared under antiviral treatment. Sicca Syndrome was observed in 17 cases $(22,36 \%)$. RM were associated to other extrahepatic manifestations of $\mathrm{CHC}$ in $69,7 \%$ of cases, notably to mixed cryoglobulinemia (MC) $(60 \%)$ and to antinuclear antibodies (21,6\%). Anti DNA, anti SSA, anti SSB, rheumatoid factor and anti CCP were absent in all cases. A partial to total amelioration of $\mathrm{RM}$ was noted in almost patients under antiviral treatment and sometimes associated to symptomatic measures. In univariate analyse, only feminal sexe and presence of MC were significatly correlated to the presence of MR.

Conclusions: During $\mathrm{CHC}, \mathrm{MR}$ are frequent, dominated by arthralgia, myalgia and sicca syndrome. Authentic arthritis are rare and constitute a diagnostic problem essentially when they inaugurate the disease. $\mathrm{MC}$ is the immunological factor the most associated with RM. Treatment of MR still antiviral.

Disclosure of Interest: None declared

DOI: 10.1136/annrheumdis-2018-eular.5886

\section{AB1050 REACTIVE ARTHRITIS DUE TO PARVOVIRUS B19 MAY BE OVERLOOKED IN ADULT RHEUMATOLOGY}

K. Hutton ${ }^{1}$, K.M. Carlsen ${ }^{2} .{ }^{1}$ Department of Dermatology, Bispebjerg University Hospital, Copenhagen; ${ }^{2}$ Copenhagen Centre for Arthritis Research, Rigshospitalet Hillerød, Hillerød, Denmark

Background: In 1991 parvovirus B19 analyses, for the 5th disease of childhood, were established in Denmark, revealing the first epidemic. Prior to this, erythema infectiosum, "slapped cheek appearance" and reactive arthritis were only clinical diagnoses. A project in the PhD thesis Human Parvovirus B19 Erythrovirus Methods established for Virological and Diagnostic Aspects 2006 APMIS 120;114:1-121, revealed that $35.4 \%$ of 3.628 pregnant women were susceptible to parvovirus B19 infection.

Objectives: To present manifestations of parvovirus B19 in a susceptible adult population

Methods: , During the years 20112014 and 2017 the following patients were admitted.

Case 1: A 61-year-old woman with rash and thrombocytopenic purpura in April 2011. Routine blood tests, RF, anti-CCP, tuberculosis, ANA and anti-ds DNA, serological tests for viral and autoimmune hepatitis were performed. Also ECG and finally, parvovirus B19 IgM and IgG, PCR for Parvovirus B19 was performed, June 2011

Case 2: A 52-year-old woman with rash, swollen joints and pericarditis in May 2011

Case 3: A 45-year-old woman with swollen joints, March 2011

Case 4: A 45-year-old woman with swollen joints and rash, November 2014

Case 5: A 68-year-old man admitted to the Department of Dermatology and diagnosed with Sweet's Syndrome, severe anaemia and swollen joints, September 2014

Case 6: A 62-year-old woman transferred due to parvovirus B19 positive IgM and IgG antibodies with reactive arthritis and itching skin rash, June 2017

All six patients were tested as mentioned above in Case 1.

Results: In all 6 patients, routine tests were normal. HBV, STD, ANA, antidsDNA, TB, RF and anti-CCP were negative. Parvovirus DNA by PCR, IgM and IgG antibodies were positive except for the last patient, who did not have PCR but a punch biopsy of her maculo-papulary rash performed. Ultrasound revealed oedema and synovitis in the patients.

The 1st patient contracted parvovirus B19 from her 4-year-old grandchild, the 2nd during work in a daycare. They both had self-limiting infections and pericarditis resolved without treatment. The 3rd patient was also infected from daycare and her persistent synovitis, required DMARDs and biologic treatment.

The 4th patient was treated with DMARDs for one year. The 5th patient had severe anaemia for $1 / 2$ year, almost overlooked due to Sweet's syndrome but his anaemia (due to replication of the parvovirus in erythroblasts) was finally diagnosed. The patient continues treatment for Sweet's Syndrome at the Department of Dermatology.

The 6th patient slowly recovered from her skin rash and reactive arthritis.

Conclusions: The 5th disease of childhood was almost overlooked in 6 patients probably due to their ages. Therefore, clinicians must be aware of the need for parvovirus B19 analyses in seronegative RA patients. Parvovirus B19 occurs as epidemics approximately every $3 r d$ year as an occupational risk, jeopardising patients' female family members who may be pregnant and develop foetal hydrops and anaemia necessitating intrauterine blood transfusion at a Foetal Medical Centre.

Disclosure of Interest: None declared

DOI: 10.1136/annrheumdis-2018-eular.7378

\section{AB1051 BRUCELLOSIS IN RHEUMATOLOGY: A STUDY OF 27 CASES IN TUNISIA}

K. Saadaoui, H. Sahli, S. Rekik, H. Ajlani, M. Mariem, T. Aissaoui, S. Boussaid, E. Cheour, M. Elleuch, on behalf of CHU LARABTA. Rheumatology, CHU LA RABTA, Tunis, Tunisia

Background: Brucellosis is an endemic zoonosis in the Mediterranean basin The osteo articular localization, although rare, remains redoubtable.

Objectives: The aim of our work is to study the characteristics of osteoarticular brucellosis in Tunisia.

Methods: This is a descriptive retrospective study including 27 cases of osteoarticular brucellosis, collected in a rheumatology department over a period of 12 years (2006-2017)

Results: The Sex Ratio Female/male was 1.07 with an average age of 50.9 years. ${ }^{15-76}$ Diagnosis delay was 5.9 months [0.3-13]. The diagnosis of spondylodiscitis was retained in 23 patients (85.2\%) and sacroiliitis in 4 cases (14.8\%). Spinal or sacroiliac pain was found in all patients. Fever was present in 22 patients sweat in 11 patients, loss of weight or appetite were respectively found in 16 and 12 patients. Eleven patient reported fatigue.

Laboratory examination found high erythrocyte sedimentation rate in 23 patients with an average of $69.4 \mathrm{~mm} .{ }^{10-120} \mathrm{CRP}$ was elevated in 26 patients with an average of $59.6 \mathrm{mg} / \mathrm{L}$ [3.3-237], the WBC average was 9451 elements $/ \mathrm{ml}$ [5550 14 000]. Wright serology was positive in all cases, Rose Bengal was positive in 15 cases, and IFI was positive in 3 cases. Brucella melitensis was isolated in blood cultures in 3 cases.

Standard X Rays showed disk involvement in 21 patients, vertebral lesions in 20 cases and sacroiliitis in 4 patients. Sectional imaging (MRI or CT) was performed for all patients confirming standard $X$ rays data and showing abscess in 13 patients and epiduritis in 7 patients.

The most affected spinal segment was the lumbar one (13 cases), cervical localization was found in 5 cases and thoracic in only 1 case. The spondylitis was multifocal in 2 cases.

Spinal disc biopsy was performed in only 4 cases, neither anatomopathologic nor bacteriological examination were conclusive.

The treatment was based on the cycline and rifampicin combination for an aver age duration of 4.5 months. ${ }^{3-9}$

The evolution was favourable in the majority of the cases with a relapse in only 2 cases.

Conclusions: Osteoarticular brucellosis is a focal form of brucellosis, the most frequent location is spinal and whose adequate treatment allows a favourable evolution.

Disclosure of Interest: None declared

DOI: 10.1136/annrheumdis-2018-eular.7350

\section{AB1052 1 COMPARISON OF SEVERAL BIOMARKERS (MMP-2, MMP-9 AND ITS INHIBITOR TIMP-1, CTX-II, CALPROTECTIN AND COMP) IN THE SYNOVIAL FLUID AND SERUM OF PATIENTS WITH AND WITHOUT SEPTIC ARTHRITIS}

M. Couderc ${ }^{1,2}$, C. Peyrode ${ }^{2}$, B. Pereira ${ }^{3}$, E. Miot-Noirault ${ }^{2}$, S. Mathieu ${ }^{1}$

M. Soubrier ${ }^{1}$, J.-J. Dubost ${ }^{1} .{ }^{1}$ Rheumatology, CLERMONT FERRAND

UNIVERSITARY HOSPITAL; ${ }^{2}$ Imost, UMR 1240/INSERM/UCA; ${ }^{3}$ biostatistic unit, CLERMONT FERRAND UNIVERSITARY HOSPITAL, Clermont-Ferrand, France

Background: Diagnosing septic arthritis (SA) is an emergency, and for a diagno sis to be made a microorganism must be found in the synovial fluid or blood culture. Besides direct bacteriological examination, which is positive in only $25 \%$ to $50 \%$ of cases, no laboratory examination can differentiate SA from other forms of arthritis.

Objectives: To assessed the performances of several serum and synovial bio markers (matrix metalloproetinase $[\mathrm{MMP}]-2$, MMP-9, the metalloproteinase inhibitor [TIMP]-1, cartilage oligomeric matrix protein [COMP], C-terminal telopeptide of type II collagen [CTX-II], and calprotectin [CALP]) for discriminating between septic and non-SA in a population of patients with suspected SA.

Methods: Using the ELISA technique, a number of potential biomarkers (MMP-2, MMP-9, TIMP-1, COMP, CTX-II, and CALP) were measured in the synovial fluid and serum of 39 patients with suspected SA (of whom 21 had SA and 18 non-SA) (. The levels of the various markers were compared on univariate and then multivariate analysis using logistic regression. 